\title{
Aberration-corrected STEM of Cross-sectional View of Core-shell Nanowires Prepared by Ultramicrotomy
}

J. $\mathrm{Xu}^{1}$ and J. Y. Liu ${ }^{2}$

1. School for Engineering of Matter, Transport and Energy, Arizona State University, Tempe, Arizona 85287, USA

2. Department of Physics, Arizona State University, Tempe, Arizona 85287, USA

The ultramicrotome is used extensively for preparing cross-sectional samples for optical and/or electron microscopy [1]. Ultramicrotomed thin sections usually have a uniform thickness and retain the original elemental distribution in phases of the sample [2]. Generally speaking, thin nanowires (NWs) are ideal for electron microscopy observations. However, it is extremely difficult, if not impossible, to examine the cross-sectional view of long NWs. Such situation arises if one wants to examine the interfacial atomic structures of core-shell nanowires or other more complicated configurations. In these cases, embedding the NWs into resins and ultramicrotoming the cured resin/NW composite provide a method to extract information about interfacial atomic structures of core-shell NWs or other types of nanostructures. We report here, via the use of ultramicrotome to prepare cross-sectional samples, the investigation of the interfacial structures of the $\delta-\mathrm{Bi}_{2} \mathrm{O}_{3}$ epitaxially grown onto the selected facets of pure wurzite $\mathrm{ZnO} \mathrm{NWs}$.

$\mathrm{Bi}_{2} \mathrm{O}_{3} / \mathrm{ZnO}$ composite nanostructures were embedded into capsule modes and polymerized for $24 \mathrm{~h}$ at $70^{\circ} \mathrm{C}$. The cured block was then trimmed to dimensions of $0.5 \mathrm{~mm} \times 0.5 \mathrm{~mm}$ and mounted on the ultramicrotome (Leica Ultracut R microtome) and sectioned with a diamond knife with a wedge angle of $45^{\circ}$. Thin slices of the sample with a thickness of 30-50nm were produced. Lacy-carbon coated TEM copper grids were used to collect the ultrathin sections. A thin layer of carbon was coated onto the ultramicrotomed thin sections prior to TEM/STEM observation. Aberration-corrected STEM, in the highangle annular dark-field (HAADF) imaging mode, was used to examine the cross-section core-shell NWs.

Figure 1a and $1 \mathrm{~b}$ show low and high magnification HAADF images of a cross-section of a $\delta-\mathrm{Bi}_{2} \mathrm{O}_{3} / \mathrm{ZnO}$ $\mathrm{NW}$ with the electron beam parallel to the $\mathrm{ZnO}$ [0001] zone axis. The side facets of the $\mathrm{ZnO}$ NWs consist of $\{10-10\}$ and $\{11-20\}$ surfaces and the $\delta-\mathrm{Bi}_{2} \mathrm{O}_{3}$ grew selectively only on the $\{11-20\}$ facets. Our previous work demonstrated that the $\delta-\mathrm{Bi}_{2} \mathrm{O}_{3}\{100\}$ facets grew epitaxially onto the $\{11-20\}$ facets of the $\mathrm{ZnO} \mathrm{NWs}$ [3]. With the analysis of the atomic arrangements of the $\mathrm{Zn}$ and $\mathrm{Bi}$ atoms as revealed in Fig. $1 \mathrm{~b}$, it is proposed that the oxygen layer of $\delta-\mathrm{Bi}_{2} \mathrm{O}_{3}$ may be in direct contact with the $\mathrm{ZnO}(11-20)$ surface. The detailed arrangement of the interfacial oxygen atoms is still unclear and is an active area of investigation. The wetting behavior between the $\delta-\mathrm{Bi}_{2} \mathrm{O}_{3}\{100\}$ and the $\mathrm{ZnO}\{11-20\}$, however, suggests strong interfacial reactions. A schematic illustration of the proposed interfacial structure is shown in Fig. 1c. Schematic diagrams in Figure 1d show the atomic arrangement of the reconstructed $\delta-\mathrm{Bi}_{2} \mathrm{O}_{3}(100)$ and $\mathrm{ZnO}$ (11-20). The interfacial $\mathrm{Bi}$ atoms are strained to accommodate the epitaxial relationship, and a good dimensional match exists between the $\mathrm{ZnO}\{11-20\}$ and the $\delta-\mathrm{Bi}_{2} \mathrm{O}_{3}\{100\}$ planes. The fact that $\delta-\mathrm{Bi}_{2} \mathrm{O}_{3}$ has a high concentration of oxygen vacancies may facilitate to form a strong bonded interface [4]. Detailed analyses of the interfacial structures and the growth mechanisms will be discussed [5].

\section{References:}

[1] H. Plummer, Microsc. Microanal. 3 (1997), p.239.

[2] L.Y. Wei and T. Li, Microsc. Res. Tech. 36 (1997), p.380. 
[3] J. Xu, J. Liu, Microsc. Microanal. 19 (2013), p.1516.

[4] J. Luo, J. Am. Ceram. Soc. 95 (2012), p.2358.

[5] This research was funded by Arizona State University. We gratefully acknowledge the use of facilities within the LeRoy Eyring Center for Solid State Science at Arizona State University.

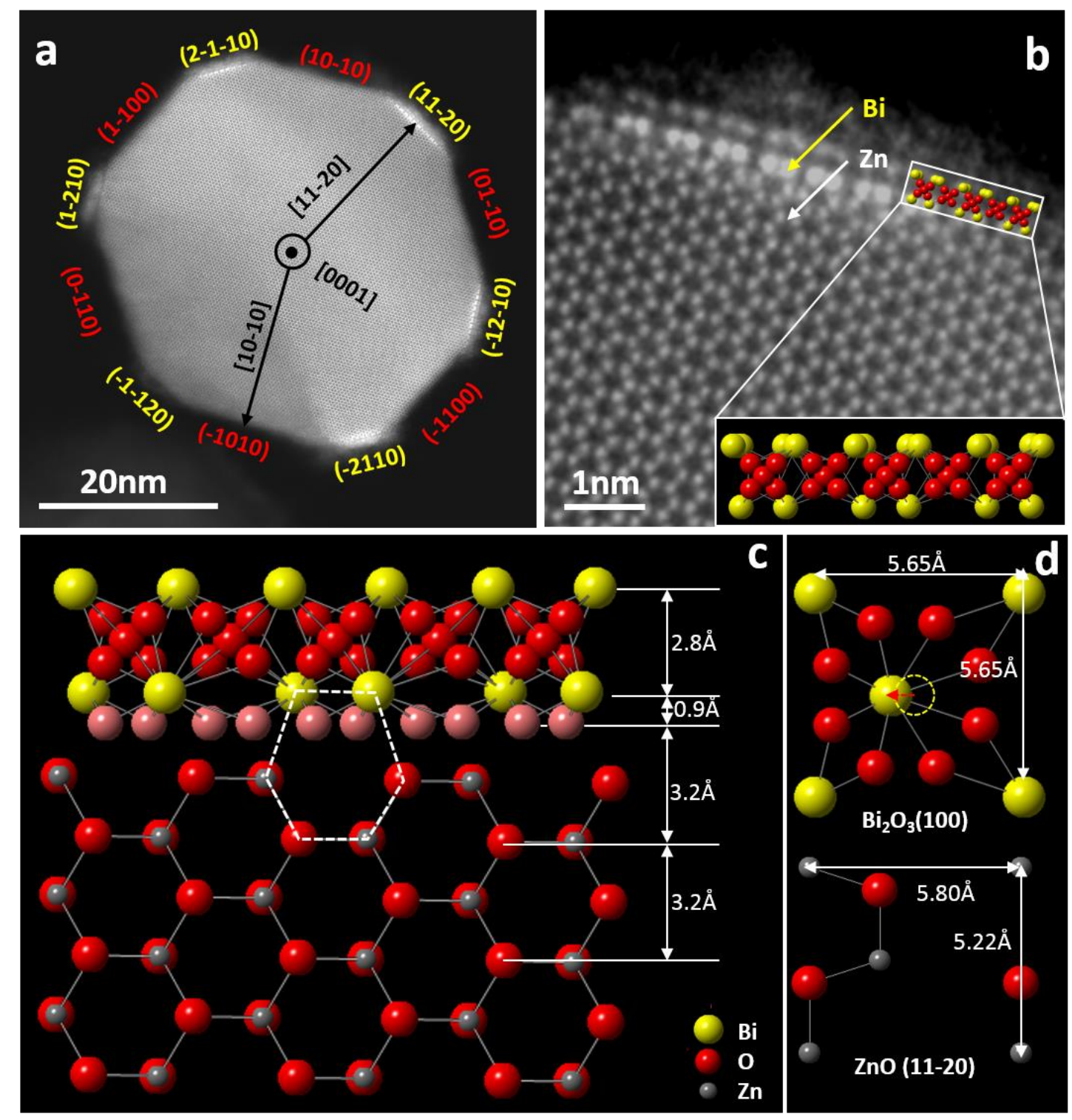

Figure 1 (a-b) Low and high magnification HAADF images of the cross-sectional view of a typical $\delta$ $\mathrm{Bi}_{2} \mathrm{O}_{3}$ decorated $\mathrm{ZnO}$ nanowire. Epitaxial layers of $\delta-\mathrm{Bi}_{2} \mathrm{O}_{3}$ were deposited selectively onto $\mathrm{ZnO}\{11-20\}$ facets. The reconstruction of the interfacial $\mathrm{Bi}$ atoms is clearly revealed in Figure $1 \mathrm{~b}$. The proposed atomic structure of the interfacial region is schematically illustrated in Figure 1c. Figure 1d shows the proposed positions of the interfacial atoms on the $\delta-\mathrm{Bi}_{2} \mathrm{O}_{3}$ (100) and $\mathrm{ZnO}$ (11-20) planes. To accommodate the epitaxial relationship, one Bi atom (indicated by the red arrow in Figure 1d) has to be shifted by $0.09 \mathrm{~nm}$ to bond directly with the $\mathrm{Zn}$ atom. 